\section{Stuart Parkin receives 2014 Millennium Technology Prize for opening big data era}

$\mathrm{T}$ echnology Academy Finland has named IBM Fellow Stuart Parkin to receive the 2014 Millennium Technology Prize in recognition of

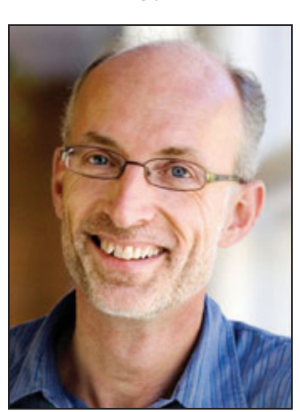
his discoveries, which have enabled a thousandfold increase in the storage capacity of magnetic disk drives. Parkin's innovations have led to a significant expansion of data acquisition and storage capacities, which in turn have underpinned the evolution of large data centers and cloud services, social networks, and music and film distribution online. Parkin is a leading innovator in the field of spintronics, which relies on the magnetic spin of electrons rather than their charge to store bits. His discoveries rely on magnetoresistive thin-film structures and the development of the giant magnetoresistance (GMR) spin-valve read head. Following the discovery of GMR in 1988, Parkin rapidly transformed this scientific observation into a practical data-storage technology. The prize recognizes technological innovation and is worth one million euros. Parkin is an IBM Fellow, consulting professor at Stanford University, director of the Max Planck Institute of Microstructure Physics, and Alexander von Humboldt Professor at Martin Luther University, Halle-Wittenberg.

\section{Kavli Prize in Nanoscience goes to Ebbesen, Hell, and Pendry}

The Kavli Prize in Nanoscience 1 is shared between Thomas W. Ebbesen, Université Louis Pasteur, Université de Strasbourg, France, Stefan W. Hell, Max Planck Institute for Biophysical Chemistry, Germany, and Sir John B. Pendry, Imperial College London, UK. They received the prize "for transformative contributions to the field of nano-optics that have broken long-held beliefs about the limitations of the resolution limits of optical microscopy and imaging." With their respective work, they have challenged established beliefs about the resolution limits of optical imaging, showing that light can interact with nanostructures smaller than its wavelength.

Thomas W. Ebbesen has shown that there can be an extraordinary transmission of light through nano-fabricated holes in thin metal films. The sizes of

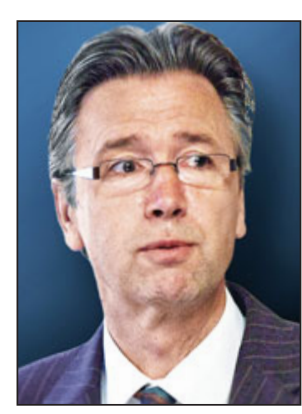

those holes are far smaller than the wavelength of the light itself. His experiments in 1998 yielded results that challenged prior accepted theories of light propagation through small holes. The underlying reasons have to do with efficient re-radiation made possible through plasmons - a cooperative oscillation of electrons, which is particularly intense in nanoscale structures. Ebbesen's understanding of the basic mechanism, and his implementation of different structures to enhance the focus, direction, and general control of the plasmonic enhancement have led to new means of increasing the efficiency, spatial focus of photonic devices, and sensitivity of optical sensors.

In optical microscopy, a key issue for image clarity has been distinguishing the signal from a broad background of noise. Stefan W. Hell showed how the back-

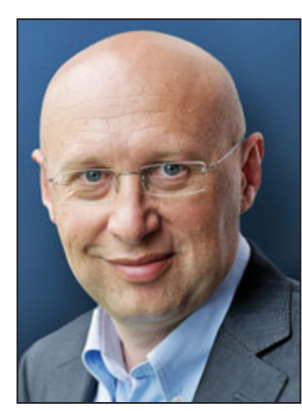
ground noise can be controlled by strategically "shutting off" molecular transitions at the appropriate time. He refers to this technique as stimulated emission depletion (STED), a technique that has now become accessible through instruments which he has helped to make commercially available. Not only has
STED enabled imaging at dimensions far smaller than optical wavelengths for a broad class of materials, it has made this a viable option for the life sciences. Hell's techniques have made possible direct observations of dynamical processes in living cells at nanoscale resolution.

Sir John B. Pendry has created a model for constructing a perfect lens, based on materials not normally found in nature or metamaterials. Such special

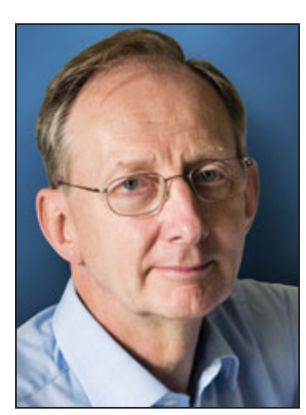

materials can have a negative index of refraction. It was Pendry's insight to reinvestigate these ideas in the context of real materials such as silver, gold, and copper, and to formulate the guidelines for the eventual realization of perfect lenses. With a growing body of experimental validation of his work, he has further stimulated a plethora of activities extending these concepts across the visible spectrum and beyond. Pendry helped to formulate rules on how to incorporate different kinds of materials (metals and dielectrics) with nanoscale structures to form larger scale metamaterials with optical properties that nature has not before provided. His work is expected to lead to dramatically improved levels of efficiency in light emission, storage, and sensing than is currently available.

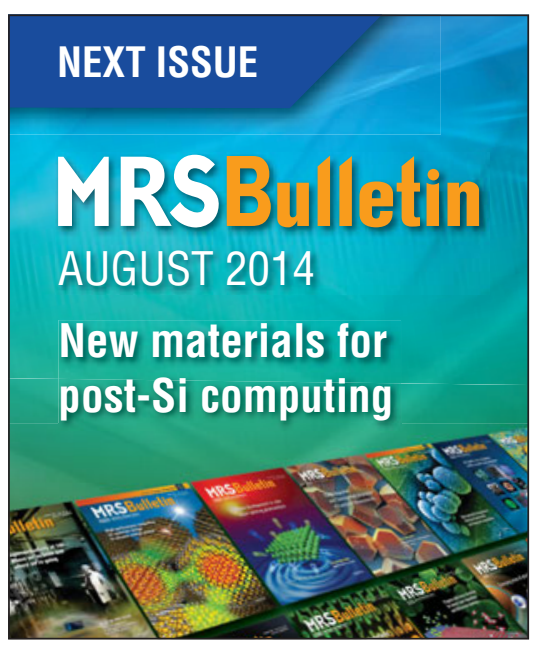

